\title{
No evidence found for anticipatory looking responses to specific satiety in adult humans.
}

\author{
Rachel Crosby, Ljerka Ostojić, Edward Legg and Nicola S Clayton
}

\author{
Department of Psychology \\ University of Cambridge
}

Cambridge CB2 3EB

United Kingdom

\section{Abstract}

Recent research has uncovered a developmental paradox within theory of mind. While spontaneous response measures indicate sensitivity to false beliefs in infants before their first birthday, tasks involving elicited response measures of false belief are only passed consistently from 4 years of age. In adults, it has been suggested that these spontaneous responses may result from a minimal theory of mind system, which allows rapid and automatic attribution of mental states to others. This minimal system trades off cognitive efficiency against flexibility, and as a result is susceptible to certain signature limits.

It has also been proposed that the limitations of the minimal system in adults may resemble the limitations demonstrated by non-human animals in tasks thought to involve mental state attribution. To date, studies investigating spontaneous responses in humans have focused on the attribution of false beliefs about location and identity. However, research into animal mental state attribution has also included studies of desire state attribution in Eurasian jays. Here we have adapted the specific satiety paradigm used with Eurasian jays to investigate adult humans' I anticipatory looking responses based on another individual's specific satiety.

Participants observed a protagonist eating a food to satiety followed by presentation of a choice between different food options. Participants demonstrated an anticipatory looking response only when a strong behavioural cue of the protagonist's desire was available: a direct verbalisation of the protagonist's satiety. This contrasted with elicited measures, as participants were able to make explicit behavioural predictions based on the protagonist's satiety, both with and without verbalisations. 
However, although no clear evidence was found for spontaneous desire attribution in this study, it is difficult to draw conclusions from these results given the small sample sizes available and the current replication failures of studies demonstrating spontaneous responses to false belief.

\section{Introduction}

Traditional false belief tasks involve directly questioning children about behavioural outcomes and are only passed consistently around the age of 4 (Wellman \& Liu, 2004). However, recent research with human infants has focused almost exclusively on spontaneous responses. These contrast with elicited-response false belief tasks in that they measure unprompted automatic responses to a scenario (Baillargeon, Scott, \& He, 2010). Tracking of eye movements has shown spontaneous looking responses to false beliefs in children as young as 10 months (Kovács, Téglás, \& Endress, 2010; Surian, Caldi, \& Sperber, 2007); while other spontaneous measures, such as motor cortex activation, have also shown indications of belief processing in the first year of life (Southgate \& Vernetti, 2014).

Research into spontaneous responses has sparked a new wave of investigation into the mechanisms underlying theory of mind, with two main interpretations emerging (Low \& Perner, 2012). Early mindreading accounts argue that spontaneous responses are indicative of a fully representative theory of mind (Baillargeon et al., 2010; Carruthers, 2013). Whereas conceptual change accounts argue that there is a qualitative difference between the social cognitive abilities possessed by infants and adults (Apperly \& Butterfill, 2009; Csibra \& Gergely, 1998; Nichols \& Stich, 2003; Ruffman, Taumoepeau, \& Perkins, 2012).

One such conceptual change account is the two systems model described by Apperly and Butterfill (Apperly \& Butterfill, 2009; Butterfill \& Apperly, 2013). This model suggests that spontaneous responses result from a minimal theory of mind system that is separate to the system that generates explicit responses. This minimal system achieves cognitive efficiency by representing inflexible belief-like registrations as opposed to full representations of beliefs. As a result, the minimal system will have certain signature limits beyond which spontaneous responses fail and the full explicit system must take over for a correct response to be produced, for example second order perspective taking (Low \& Watts, 2013), although c.f. (Kulke, von Duhn, Schneider, \& Rakoczy, 2018).

Apperly and Butterfill have suggested that there may be some similarities between human minimal theory of mind and the mechanisms used by non-human animals to solve tasks involving mental state attribution (Apperly \& Butterfill, 2009; Butterfill \& Apperly, 2013). In reference to theory of mind in infants and animals, they state that "there is evidence that these abilities are limited and limited in similar ways" (pg. 958, Apperly and Butterfill 2009). In their original paper they specifically 
discuss gaze-following and perspective-taking in chimpanzees as well as caching behaviour in scrub jays. In further support of this, subsequent research has shown that chimpanzees produce spontaneous anticipatory looking responses to false belief tasks, perhaps suggesting that some form of minimal theory of mind system may be conserved between apes and humans (Krupenye, Kano, Hirata, Call, \& Tomasello, 2016, 2017).

Currently no human research has investigated spontaneous responses to desires or desire-like states. This contrasts with research into theory of mind-like abilities in non-human animals, which has included both belief-attribution in chimpanzees and desire-attribution in Eurasian jays (Call \& Tomasello, 2008; Ostojić, Shaw, Cheke, \& Clayton, 2013). Eurasian jays' co-operative social cognitive abilities have been investigated by utilising their natural food sharing courtship behaviour, in which males give food directly to their female partner (Ostojić et al., 2014, 2013; Ostojić, Legg, et al., 2016; Ostojić, Cheke, Shaw, Legg, \& Clayton, 2016). The female's desire-state was manipulated via specific satiety, a sensory phenomenon whereby continued consumption of a single food causes a temporary decline in the desirability of that food (Balleine \& Dickinson, 1998; Havermans, Janssen, Giesen, Roefs, \& Jansen, 2009). It was found that the males shifted their pattern of food sharing in line with the females' desires, such that they shared a greater proportion of waxworms with their partner when she was pre-fed mealworms than when she was pre-fed waxworms (Ostojić et al., 2013).

In order to investigate the similarities between human minimal theory of mind and desire attribution in non-human animals, the present study adapted the specific satiety paradigm used with Eurasian jays to test the spontaneous responses of adult humans to others' desires. In the Eurasian jay paradigm, the females were pre-fed either waxworms or mealworms, followed by a food sharing test phase during which the male had the option of sharing up to 20 waxworms or mealworms with the female. To modify this for adult humans, participants watched videos of a protagonist eating either apples or crackers, followed by a video clip in which the protagonist was offered a subsequent choice between apples and crackers. If the participants are able to spontaneously process the protagonist's choices based on their inferred satiety they are expected to direct their anticipatory looking towards the non-sated food. If no such directed anticipatory looking is found this may weaken the argument that theory of mind-like abilities in non-human animals are equivalent (or highly similar) to a minimal theory of mind system in humans. 


\section{Experiment 1}

\subsection{Participants}

We chose a minimum sample size of 25 participants as this was slightly larger than those used in previous studies that have identified anticipatory looking responses in adults (19-20) (Low \& Watts, 2013; Schuwerk, Vuori, \& Sodian, 2015; Senju, Southgate, White, \& Frith, 2009). To achieve this we recruited 35 adults, 10 of these were excluded due to software error (1), experimenter error (i.e. sound was off, 2) or a lack of eye movements (i.e. looking straight ahead throughout the test, 7). This left 25 participants, 20 female, mean age 25 years 6 months, age range 20 years 8 months to 39 years 8 months. Participants were recruited through online and poster advertisements and paid $£ 4$ for taking part in the experiment. After reading an information sheet, all participants gave written consent. This experiment was approved by the Department of Psychology Ethics Committee (PEC) at the University of Cambridge.

\subsection{Procedure}

Participants were tested in the Department of Psychology at the University of Cambridge, between July and November 2016. Participants were seated in front of a 27-inch screen allowing unconstrained viewing at a distance of approximately $1 \mathrm{~m}$. To allow gaze direction to be determined participants were recorded during the trial using a Logitech webcam and Camtasia software. All participants were presented with two conditions (an apples condition and a crackers condition), and their responses to the two conditions were compared in a within-subject design.

Each condition involved a video being presented to the participant. The videos comprised two sections: an eating clip (Figure 1a) and a choice clip (Figure 1b). The eating clip showed a protagonist eating from a bowl of food: apple slices in the apples condition and Ritz crackers in the crackers condition. The choice clip was identical between the two conditions: the protagonist was seated between two bowls, one bowl containing apple slices and the other containing Ritz crackers. The order of presentation of the two conditions was counterbalanced across participants.

To prevent carryover effects the videos were captioned 'Day 1 ' before the first video and 'Day 2' before the second. This procedure was chosen to clarify for the participants that the videos were depicting the protagonist's actions on two separate days, and thus the protagonist's satiation state in the second video should be independent of the first video. 

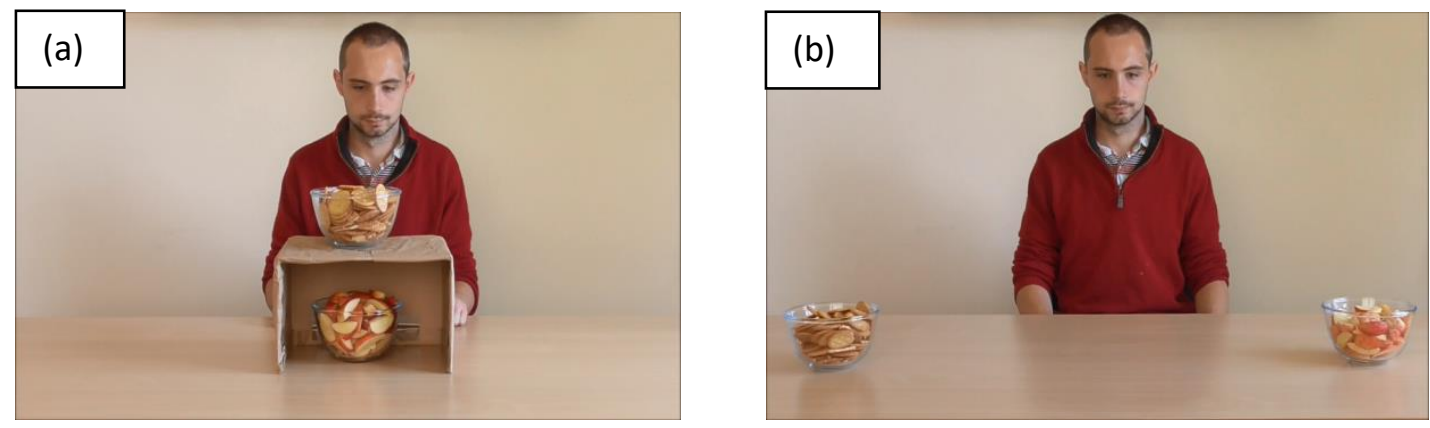

Figure 1: Stills taken from the videos showing the male protagonist. Throughout the four minute eating clip (a) the protagonist was shown eating either crackers or apples; the other food-type was hidden below a box so it was visible to the participant but not the protagonist. In the choice clip (b) the protagonist was presented with separate bowls of the two different food-types.

Each eating clip was four minutes in length, as a previous study found this to be long enough for participants to identify a change in the protagonist's desire-state (Legg, 2014). Food items were eaten at a constant rate of one item every 15 seconds, and protagonists maintained a neutral expression throughout. The apples were cut into slices of a similar size to the Ritz crackers, and both food items took approximately the same amount of time to eat.

A beep occurred each time the protagonist reached towards the bowl, so that the participant would learn to expect a reaching movement when the beep was played. To prevent participants from visually habituating to the presence of one food during the eating clip, the other food was placed under a box with an open-side facing the participant. The bowl was therefore visible to the participant but not visible to the protagonist (Figure 1a). This ensured that any difference in looking preference towards the non-sated food during the choice clip could not be explained by perceptual novelty. As the other bowl of food was not visible to the protagonist it should not appear to the participant as if they were choosing one food over the other.

After watching the 4-minute eating clip, participants were immediately presented with the choice clip. In this, the protagonist entered the room and sat facing the participant with two bowls - one containing apples and one containing crackers - on a table in front of them (Fig. 1b). In order to increase the chances of an anticipatory looking response, the beep used in the eating clip was repeated. This is analogous to the light and tone used in spontaneous false belief studies to cue participants to anticipate a reaching movement (Senju, Southgate, Snape, Leonard, \& Csibra, 2011; Senju et al., 2009; Southgate, Senju, \& Csibra, 2007). The protagonist then leant forward in a 1 second clip and the video was paused just before the protagonist would have reached for the bowl, displaying the final image statistically for 5 seconds (Senju et al., 2009). The two bowls were placed 
as far apart as possible so that eye movements would be easier to code, and the positions of the two foods were counterbalanced across participants.

\subsection{Recording of responses}

The participants' spontaneous responses were measured by coding the video recordings after the trials had concluded. For each participant two measures of anticipatory looking were assessed from the same recordings: (i) the initial direction in which the participant looked, (ii) the total time spent looking towards each bowl of food (Low \& Watts, 2013; Schuwerk et al., 2015; Senju et al., 2009). Frame-by-frame video was exported into VLC player and looking directions were recorded through experimenter observation. $20 \%$ of videos (5 out of 25 ) were coded by a second experimenter who was blind to condition. Inter-rater reliability was high, with $96 \%$ agreement over the occurrence of looks. The direction of first looks provided 100\% agreement, and the difference between experimenters' measurements of looking time to each food-type was just $8.1 \%$ on average $(0.050 \pm$ 0.048 seconds, mean \pm standard deviation).

At the end of the experiment participants' explicit responses were recorded by asking them to mark their expectation for the protagonist's choice in each video along a line from apples to crackers (Figure 2), giving a quantitative representation of their prediction. The position of the choices (left and right) was randomised and counterbalanced between participants. This continuous response mirrored the continuous nature of the looking time measure and allowed participants to give an intermediate answer if they were not confident of which food the protagonist would choose. The explicit questions were given to the participant after the presentation of both of the videos to prevent their explicit answers from influencing their eye movements.

$$
\text { Apples }
$$

Crackers

Crackers Apples

Figure 2: The lines as they appeared to the participant when giving their explicit answer. Participants were given lines in one of the two orders, apples to crackers or crackers to apples. Each participant was presented with two lines, one for 'Video 1 ' and one for 'Video 2'. The explicit question for both conditions was given to participants after they had watched the second video. 


\subsection{Analysis}

To analyse the participants' understanding of the protagonist's desire-state, a within-subject comparison was made between each participant's anticipatory looking responses in the two conditions; apples and crackers. Previous studies looking at spontaneous responses to false belief tasks have typically compared looking responses to 'chance' (usually defined as 0.5 ), assuming that uninformed participants are equally likely to look to each location. However, this does not account for baseline variation in looking preferences across participants. By comparing across two trials it was possible to control for disparities in looking times across locations due to visual differences in colour and texture, personal preference for either food, handedness, or other underlying side biases. This should also prevent differences in eye movements from being affected by salient visual features of the scenario as they should be constant across conditions (Kulke et al., 2018). When the difference between the two conditions is measured this should extract only the variation resulting from anticipation of the protagonist's choice. This also reflects the analysis used in desire-state attribution experiments with Eurasian jays, in which the male's food sharing was compared in a within-subject design between a pre-fed waxworms condition and a pre-fed mealworms condition, in order to control for variations in the males' baseline preferences for sharing different foods (Ostojić et al., 2013).

The pattern of first looks was compared between the two conditions (apples and crackers) using a McNemar's test for consistency of responses. The proportion of time spent looking towards apples out of total time spent looking at apples and crackers $^{1}$ was compared between the two conditions using a Wilcoxon signed-rank test with corrections for ties. If participants were able to spontaneously anticipate the protagonist's choice of the non-sated food, they should spend a higher proportion of time looking at apples in the crackers condition than the apples condition.

Participants' explicit answers were also analysed using a within subject design as they may also be influenced by the participant's own baseline preference for each food type. The answers were scored numerically by measuring along the line from apples to crackers, with an accuracy of $0.05 \mathrm{~cm}$. Their answers were compared between the two conditions using a Wilcoxon signed-rank test. If participants were able to explicitly predict the protagonist's choice of the non-sated food, they should have marked closer to apples for the crackers condition than for the apples condition.

\footnotetext{
${ }^{1}$ The proportion of total looking time directed towards apples was derived using the formula $\frac{A}{A+C}$, where $A$ is the total time spent looking towards apples and $\mathrm{C}$ is the total time spent looking towards crackers. The proportion of total looking time directed towards crackers is the inverse of this value and therefore does not alter the output of the analysis.
} 


\subsection{Results and Discussion}

In response to the explicit question, participants indicated that the protagonist was more likely to choose apples after having eaten crackers than after having eaten apples (Wilcoxon signed-rank test, $n=25, V=234, p=0.015$, Cohen's $d=0.566$ ). In contrast, participants' eye movements (spontaneous measure) showed no directed response to the protagonist's specific satiety. Participants showed no difference in looking times towards apples between conditions (Wilcoxon signed-rank test, $n=25$, $V=121, p=0.621$, Cohen's $d=0.113$, Figure 3 ), and were equally likely to direct their first look towards apples in both conditions (McNemar's test, $n=25, X^{2}=0.1, p=0.752$, Figure 3 ). These results suggest that participants did not spontaneously process specific satiety despite making explicit predictions that the protagonist would choose the non-sated food. 
Experiment 1
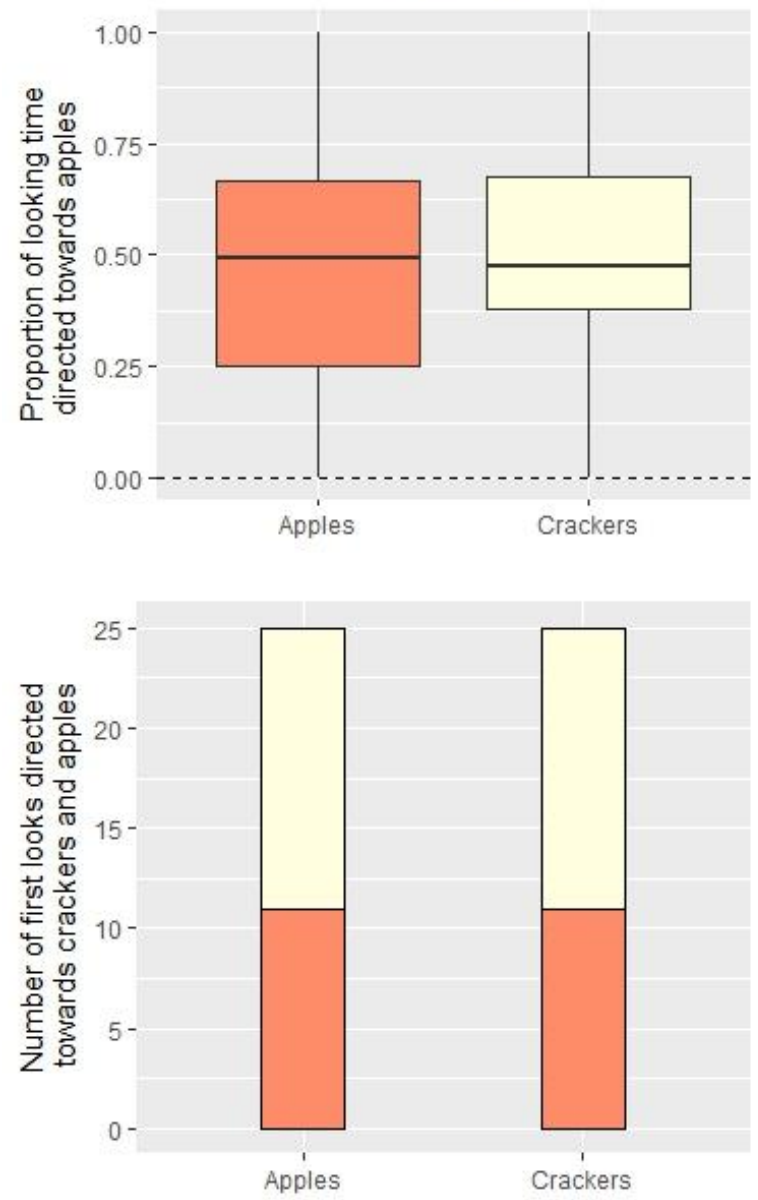

Experiment 2
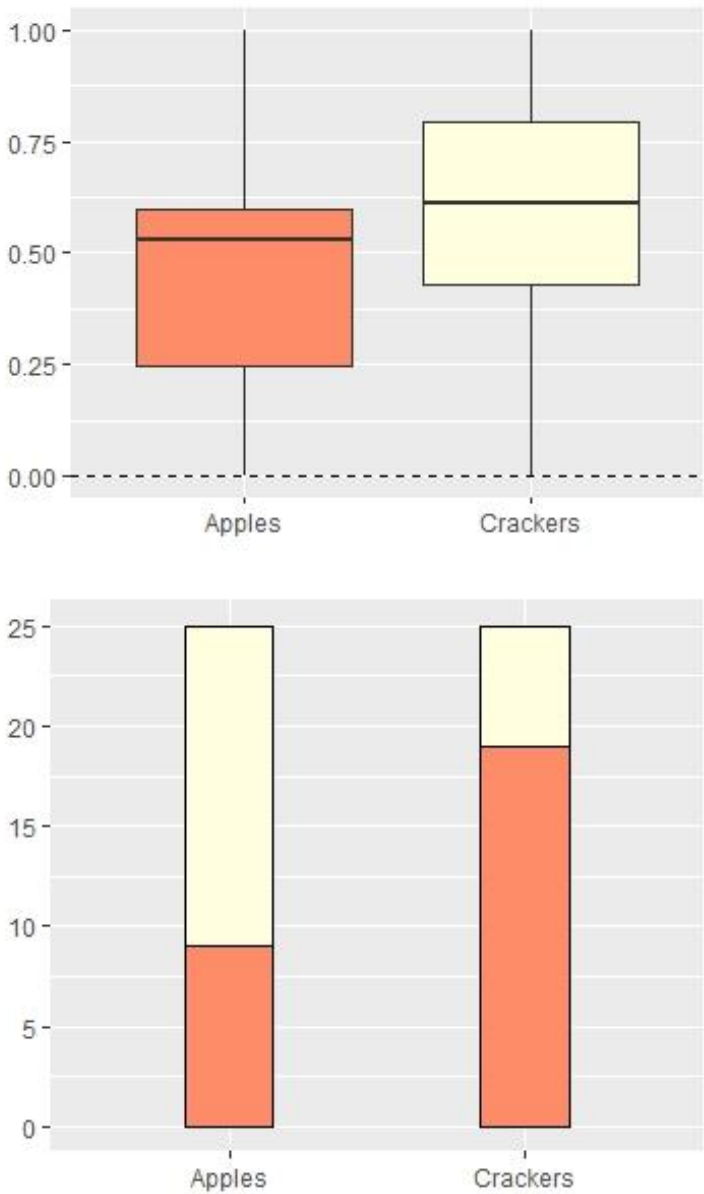

Experiment 3
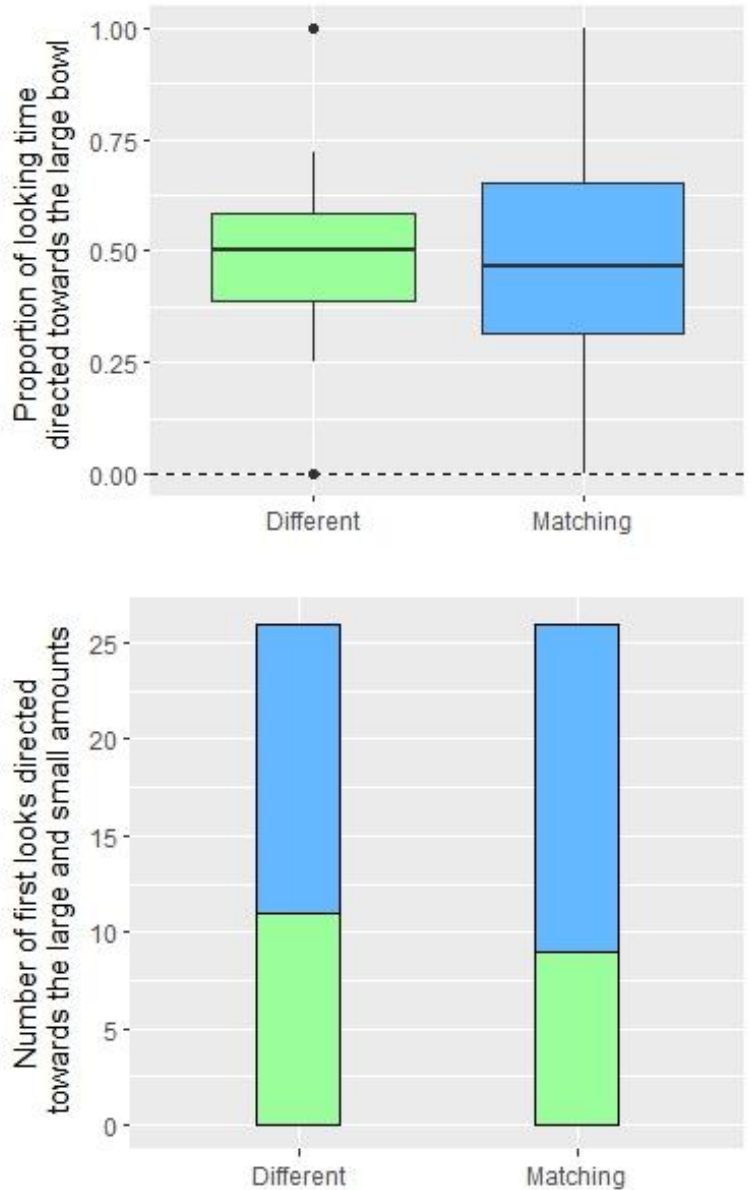

Figure 3: Spontaneous responses of participants to videos: The boxplots show looking time measures for the two conditions, apples eaten (red) and crackers eaten (yellow) for Experiments 1 and 2, different protagonist (green) and matching protagonist (blue) for Experiment 3. Boxes represent interquartile range and median (thick line), while whiskers represent the full range of the data. The barplots show the direction of first looks towards apples (red) and towards crackers (yellow) for Experiment 1 and 2 , and towards the large amount (green) and the small amount of chocolate (blue) for Experiment 3. 


\section{Experiment 2}

Subsequent to Experiment 1, we conducted a further experiment to investigate whether adults are able to spontaneously respond to specific satiety when provided with a direct behavioural cue to the protagonist's desire-state. One key difference between the Eurasian jay study and the paradigm used in Experiment 1 is that we were able to exercise some control over behavioural indications of the protagonist's satiety. That is, by instructing the actor to keep a neutral expression throughout and by timing the intervals between reaches so that food was consumed at a constant rate we limited cues that could indicate satiety. This contrasts with the Eurasian jay study, in which the female was given free access to the pre-fed food for 15 minutes, making it possible that she demonstrated behavioural indications of her satiety during this time. As a result, the males' shift in sharing pattern may be due to behaviour reading of the female's satiety during the pre-feeding phase, as opposed to true desire-state attribution.

In Experiment 2 the paradigm was modified to include a direct behavioural indication of the protagonist's desire-state: a verbalisation, "I'm full of apples/crackers" was added at the end of the choice clip (Legg, 2014). This should allow the participant to predict the protagonist's goal without needing to attribute a desire. It is highly likely that adults will have previously encountered scenarios in which other individuals (or they themselves) have said they are full of a food and subsequently chosen not to eat it. Thus, the verbalisation provides the potential to bypass direct desire-state attribution, allowing participants to predict the protagonist's choices through previous experience. If participants are able to anticipate the protagonist's choice when their satiety is verbalised this may support the suggestion that the results of Experiment 1 are due to a lack of spontaneous desire attribution.

\subsection{Participants}

Participants were 36 adults, 11 of these were excluded due to experimenter error (2), software error (4), participant not watching videos to completion (1), or a lack of eye movements (4). As for Experiment 1, 35 participants were tested initially with a further participant added to reach a minimum sample size of 25 participants after exclusion. Of these 25, 15 were female and the mean age was 25 years 3 months, with an age range of 19 years 2 months to 39 years 8 months. Participants were recruited through online and poster advertisements and paid $£ 4$ for taking part in the experiment. After reading an information sheet, all participants gave written consent. This experiment was approved by the Department of Psychology Ethics Committee (PEC) at the University of Cambridge. 


\subsection{Procedure and analysis}

Participants were tested in the Department of Psychology at the University of Cambridge, between July and November 2016. The procedure was the same as for Experiment 1 except that the desirestate of the protagonist was made overt through the addition of a verbalisation at the end of each of the videos: "I'm full of apples" or "I'm full of crackers", for the apples and crackers conditions respectively. The analyses were identical to Experiment 1. 20\% of videos (5 out of 25 ) were coded by a second experimenter who was blind to condition. The inter-rater reliability was very high, with $100 \%$ agreement for both the occurrence and direction of looks. The difference between experimenters' measurements of looking time to each food-type was just $5.0 \%(0.035 \pm 0.049$ seconds).

\subsection{Results and Discussion}

As in Experiment 1, participants explicitly answered that the protagonist was more likely to choose apples after eating crackers than after eating apples (Wilcoxon signed-rank test, $n=25, V=279$, $p=0.001$, Cohen's $d=0.809$ ). Participants also did not direct a different proportion of their total looking time towards apples in the two conditions (Wilcoxon signed-rank test, $n=25, V=105, p=0.208$, Cohen's d=0.307, Figure 3).

However, in contrast to Experiment 1, the direction of participants' first looks was dependent on what the protagonist had eaten. Participants were more likely to look first towards apples after the protagonist had eaten crackers than after the protagonist had eaten apples (McNemar's test, $n=25$, $X^{2}=5.79, p=0.0162$, Figure 3 ). These results suggest that the addition of a verbalisation of the protagonist's desire-state may have allowed participants to spontaneously anticipate the protagonist's choice.

The lack of a significant difference between the conditions in the looking time measure may be a result of the relatively small sample size used in this experiment, as well as the relative crudeness of the measure compared to other studies that were able to employ an eye tracker to measure looking time. However, it is also possible that the lack of consistency in the results between the spontaneous measures is due to a chance false positive finding in the first looks measure.

\section{Experiment 3}

One possible explanation for the results seen in Experiment 1 is that when no specific verbalisation was made the participants spontaneously attributed a general satiety for both foods. If this is the case then no food choice would be predicted as the protagonist would be expected to have a generalised satiety towards both foods. The contrast between the spontaneous responses and the 
explicit questioning could be explained by the question prompting the participants to predict that the protagonist would choose at least one of the two foods. In Experiment 2, the addition of the behavioural cue (verbalisation) may have bypassed this general satiety issue as only one food type was named, making the specificity of the satiety manipulation more obvious.

In order to investigate this possibility, in Experiment 3 we tested whether participants would be able to spontaneously respond to the protagonist's satiety for a single food type. If participants are able to spontaneously attribute general satiety they should anticipate that a sated protagonist would choose a small amount of food over a large amount of food.

\subsection{Participants}

Participants were 35 adults, 9 of these were excluded due to experimenter error (2), participant not watching videos to completion (1), or a lack of eye movements (6). This left 26 participants, 17 female, mean 23 years 3 months, age range 18 years 8 months to 41 years 0 months. Participants were recruited through online and poster advertisements and paid $£ 4$ for taking part in the experiment. After reading an information sheet, all participants gave written consent. This

experiment was approved by the Department of Psychology Ethics Committee (PEC) at the University of Cambridge.

\subsection{Procedure and analysis}

Participants were tested in the Department of Psychology at the University of Cambridge, between November 2017 and January 2018. Due to software errors in Experiment 1 and 2 caused by the high processing requirements of the Camtasia software, in Experiment 3 participants were recorded with a GoPro camera in addition to the Logitech webcam. Videos were presented to the participants in the same manner as in Experiments 1 and 2. Each video consisted of two sections: an eating clip in which a sex-matched protagonist ate a large bowl of chocolate, and a choice clip in which a sexmatched protagonist was seated between two bowls, one containing a small amount and one containing a large amount of chocolate.

Each eating clip was four minutes in length, with chocolate squares eaten at a constant rate of one every 12 seconds and protagonists maintaining a neutral expression throughout (Fig. 4a). The bowl contained 20 squares of chocolate so that the bowl was empty at the end of the 4 minute period. This was important to increase the likelihood that participants would expect the protagonist to choose more chocolate during the choice clip. If chocolate remained in the bowl at the end of the eating period this may indicate a rejection of the food, and thus it might appear unlikely to the participant that the protagonist would choose to subsequently eat more chocolate. 
As in Experiments 1 and 2, a beep occurred each time the protagonist reached towards the bowl, so that the participant would learn to expect a reaching movement when the beep was played.

In the choice clip, the protagonist entered the room and sat facing the participant with two bowls one containing 20 squares and one containing 5 squares of chocolate - on a table in front of them (Fig. 4b). In order to cue the protagonist's anticipatory looking, the beep used in the eating clip was repeated. The protagonist then leant forward in a 1 second clip and the video was paused for 5 seconds just before the protagonist would have reached for the bowl. The two bowls were placed as far apart as possible to increase accuracy of coding, and the contents of the two bowls were counterbalanced across participants.
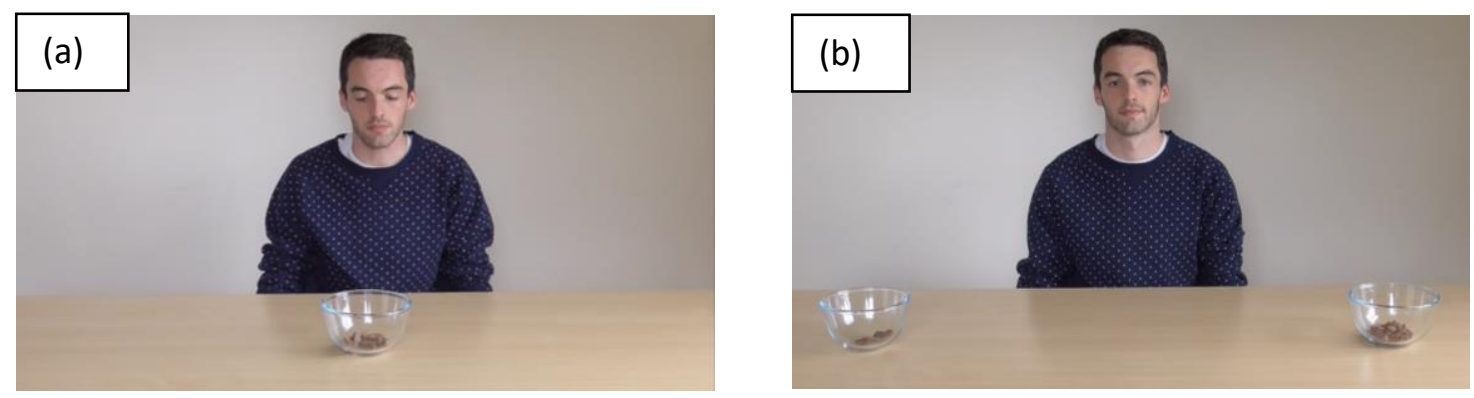

Figure 4: Stills taken from the videos showing the male matching protagonist. Throughout the four minute eating clip (a) the protagonist was shown eating chocolate pieces until the bowl was empty. In the choice clip (b) the protagonist was presented with separate bowls, one containing a large amount of chocolate, and the other a small amount of chocolate.

A pre-test was conducted on the online platform Prolific using stills from the choice section of the video to determine which bowls participants would expect an individual to choose to eat when sated or not sated. Participants from the pre-test were not used in the experiment. As expected, participants answered that they would choose to eat the large amount themselves (Wilcoxon signed-rank test, two-tailed, $n=36, p<0.01$ ) and that they would expect someone else to choose the large amount $(p<0.01)$, but that they would expect someone who had already eaten a large amount of chocolate to choose the smaller amount $(p<0.05)$.

In the main experiment, participants' responses were compared in a within-subject design, between two different conditions: a matching condition and a different condition. A within-subject design was chosen here for the same reasons as outlined above in Experiment 1, namely to eliminate looking differences due to factors other than the difference in satiety (e.g. visual differences between the small and large bowls of chocolate). The two conditions differed only in the identity of the protagonist. In the matching condition participants watched an eating clip featuring protagonist A 
eating chocolate, followed by a choice clip featuring protagonist $A$. In the different condition participants watched an eating clip featuring protagonist B eating chocolate, followed by a choice clip featuring protagonist $A$. The order of presentation of the conditions was counterbalanced across participants. The identities of protagonist $A$ and protagonist B were kept consistent across participants.

It was predicted that when participants saw a protagonist who had already eaten (matching condition) they would expect them to choose the smaller amount, but when they saw a novel protagonist being given the choice (different condition) they would expect them to choose the larger amount. Comparing between these two conditions, with identical choice clips, should allow extraction of only those eye movement differences that are related to the identity/satiety of the protagonist.

Participants' responses were recorded in the same way as in Experiments 1 and 2. Spontaneous responses were coded from the video recordings after their trials had concluded. Explicit responses were recorded by asking participants to mark their expectation for the protagonist's choice in each video along a line from small to large. The answers were scored numerically by measuring along the line with an accuracy of $0.05 \mathrm{~cm}$. The position of the choices was counterbalanced between participants. Analyses were conducted in the same way as in Experiments 1 and 2, comparing responses between the matching and different conditions in a within-subject design. 19\% of videos (5 out of 26) were coded by a second experimenter who was blind to condition. The inter-rater reliability was very high, with $100 \%$ agreement over the occurrence and direction of looks. The difference between experimenters' measurements of looking time to each food-type was just $6.7 \%$ on average (0.046 \pm 0.102 seconds).

\subsection{Results and Discussion}

Participants indicated that the protagonist was more likely to choose the small amount when the protagonist was sated (matching condition) than when the protagonist was non-sated (different condition) (Wilcoxon signed-rank test, $n=26, V=97.5, p=0.047$, Cohen's $d=0.415$ ). Across the two conditions, participants showed no difference in the proportion of total looking time directed towards the small amount (Wilcoxon signed-rank test, $n=26, V=176, p=0.731$, Cohen's $d=0.035$, Figure 3), and no difference in first look direction (McNemar's test, $n=26, X^{2}=0.1, p=0.752$, Figure 3 ). Thus, the eye movements of participants showed no sensitivity to the identity of the protagonist in the eating video (matching or different), despite showing an explicit response in line with the protagonists' satiety. 


\section{General Discussion}

Across three experiments we tested whether adults would show spontaneous anticipatory looking towards the food that a sated protagonist would desire. In all three experiments participants were able to make explicit predictions based on the protagonist's satiety. However, the experiments appeared to produce different spontaneous responses. In Experiment 2, which included a direct verbalisation of the protagonist's satiety "I'm full of apples/crackers", participants seemed able to spontaneously anticipate the protagonist's choice in one of the two measures. In contrast, in Experiments 1 and 3, where participants had no overt cues about the protagonist's desire-state, anticipatory looking did not appear to be influenced by the protagonist's satiety.

This lack of an anticipatory response in Experiments 1 and 3 is unlikely to be due to a failure of the choice clip to trigger anticipatory looking, given that a directed response was found in Experiment 2. Furthermore, no anticipatory response was found when the paradigm was reduced to one food type in Experiment 3. This suggests that the lack of anticipation in Experiment 1 is unlikely to be explained by attribution of generalised satiety to both foods.

Together these three experiments find no evidence for a spontaneous response to others' satiety in adult humans in the absence of a behavioural cue. This conclusion may appear surprising given the success of Eurasian jays in a similar specific satiety paradigm. Apperly and Butterfill have suggested that their minimal system, put forward to describe spontaneous responses in young infants and adults, may also resemble the strategy used by non-human animals to solve tasks that appear to involve mental state attribution. The experiments described here find no evidence for a shared system underlying human's spontaneous responses and Eurasian jays' food sharing patterns. However, no clear conclusions can be drawn from these results for a number of reasons.

Firstly, the paradigm used here had large differences in the behavioural cues available to the human participants relative to those available to the Eurasian jays in the original paradigm. During the prefeeding phase of the jay experiments, the behaviour of the female may provide cues to the male regarding her satiety. In Ostojić et al.'s experiment the male was found to only shift his pattern of food sharing when he was able to watch the female eating (seen condition), and not when his visual access to her was blocked during pre-feeding (unseen condition) (Ostojić et al., 2013). This control showed that the female's behaviour during food sharing alone was not sufficient to change the male's sharing pattern. However, it does not rule out an effect of differences in the female's behaviour during pre-feeding. It is possible that as the female becomes sated on the pre-fed food her eating behaviour changes in some way (e.g. slower rate of eating), providing an observable indicator of her internal state. 
Thus, the male jays may be able to utilise a behaviour reading strategy in order to determine which foods to share, rather than requiring true desire-state attribution. In contrast, in the human paradigm, we tried to minimise the cues available to the participant by keeping the expression of the protagonist neutral and the rate of eating constant. As behavioural rule accounts can be constructed post hoc it is always possible to explain the outcome of any paradigm with a behavioural rule (Lurz, 2009). However, where obvious behavioural cues are minimised the effect size of any anticipatory response may be reduced, making it much more difficult to identify an effect in the human paradigm than the jay paradigm.

Secondly, since these experiments were conducted, a series of studies have been published that indicate that evidence for spontaneous responses to false beliefs is less strong than previously believed (Kulke \& Rakoczy, 2018). A range of replication studies have failed to find evidence for the spontaneous responses previously identified in young infants (Burnside, Ruel, Azar, \& Poulin-Dubois, 2017; Dörrenberg, Rakoczy, \& Liszkowski, 2018; Kulke, Reiß, Krist, \& Rakoczy, 2017; Kulke et al., 2018). This suggests that either these spontaneous responses are artefacts of certain experimental designs or that the effect sizes are much smaller than previous studies have implied. As such, the pre-existing body of research may need questioning, and previous accounts of the mechanisms underlying spontaneous responses re-evaluating.

Finally, one of the most significant supports for Apperly and Butterfill's two system account has been the identification of certain signature limits that are theoretically predicted by their minimal model. The primary example of this is the inability of the minimal system to recognise the way in which an object appears to an individual, i.e. second order perspective taking (Low \& Watts, 2013). However, recent criticisms have been made of the original study in which this signature limit was tested. Low and Watts study compared infants' spontaneous responses in a traditional object-location test and a novel object-identity test probing second order perspective taking. Kulke et al. were initially able to replicate the results of the object-location test, but they suggested that this outcome may be due to salient visual cues during the videos (Kulke et al., 2018). In a further replication that included control measures, they found that participants' looking responses were significantly confounded with the direction of the protagonist's movements. Thus, the original differences in spontaneous responses between the object-location and object-identity tests could potentially be explained by factors unrelated to their perspective taking requirements. As such, the evidence for signature limits that align with Apperly and Butterfill's two systems account may be less robust than previously believed. Furthermore, the major support so far for Apperly and Butterfill's suggestion of the similarity between minimal theory of mind and animal mental state attribution is provided by a recent demonstration of anticipatory looking behaviour in apes (Krupenye et al., 2016). However, some 
criticisms have been made of the interpretation of these experiments. Heyes has argued that the results could be explained by 'submentalising', whereby characteristics of the stimuli cue attention in such a way that spontaneous responses are generated without any requirement for mindreading (Heyes, 2017). In order to test for such an explanation Heyes' suggested a non-social control condition, in which actors are replaced by inanimate stimuli. In response to this, Krupenye et al. conducted a further study in which they used non-social stimuli in the videos shown to the apes. In this study they found no evidence for directed anticipatory looking towards the box that a green shape should 'believe' a stone to be in. From this they concluded that there was no evidence that submentalising was responsible for their results in their original experiment.

However, this conclusion may be too strong given that the non-social stimuli actually did show a trend for more looks in the direction that aligned with the beliefs, although this trend was not significant. This is particularly important here as the study did not directly test for a difference between the original anticipatory looking paradigm and the modified non-social paradigm. It is incorrect to infer that a significant result will always differ from a non-significant result (Gelman \& Stern, 2006)., particularly as the results from the non-social condition were in the same direction as the original study. In order to test whether there was a true difference an experiment in which apes underwent both conditions would be required.

Overall, the current issues faced by comparative cognition, and psychology as a whole, regarding low sample size, low power and failures to replicate have had a significant impact on confidence in previous anticipatory looking findings. Given the issues outlined above, it would be difficult to draw any solid conclusions from the experiments presented here. Although this study may lead to interesting questions regarding desire-state attribution in humans and Eurasian jays, for any inferences to be made the experiments would need to be repeated with a much larger sample size. Thus, while current evidence suggests that any claims by Apperly and Butterfill of the similarity between human and animal theory of mind may be premature, further research is required.

\section{Acknowledgements}

Thanks for help with inter-observer reliability go to Katharina Brecht (Experiments 1 and 2) and Lovre Culina (Experiment 3).

\section{Supplementary Information}

Further information related to this study can be found on OSF at https://osf.io/94ner/, including data, documents given to participants and an example testing video. The importable data is also available at Zenodo with the DOI 10.5281/zenodo. 2525283 . 


\section{References}

Apperly, I. A., \& Butterfill, S. A. (2009). Do humans have two systems to track beliefs and belief-like states? Psychological Review, 116(4), 953-70. http://doi.org/10.1037/a0016923

Baillargeon, R., Scott, R. M., \& He, Z. (2010). False-belief understanding in infants. Trends in Cognitive Sciences, 14(3), 110-8. http://doi.org/10.1016/j.tics.2009.12.006

Balleine, B. W., \& Dickinson, A. (1998). The role of incentive learning in instrumental outcome revaluation by sensory-specific satiety. Animal Learning \& Behavior, 26(1), 46-59. http://doi.org/10.3758/BF03199161

Burnside, K., Ruel, A., Azar, N., \& Poulin-Dubois, D. (2017). Implicit false belief across the lifespan: Non-replication of an anticipatory looking task. Cognitive Development. http://doi.org/10.1016/J.COGDEV.2017.08.006

Butterfill, S. A., \& Apperly, I. A. (2013). How to Construct a Minimal Theory of Mind. Mind \& Language, 28(5), 606-637. http://doi.org/10.1111/mila.12036

Call, J., \& Tomasello, M. (2008). Does the chimpanzee have a theory of mind? 30 years later. Trends in Cognitive Sciences, 12(5), 187-92. http://doi.org/10.1016/j.tics.2008.02.010

Carruthers, P. (2013). Mindreading in Infancy. Mind \& Language, 28(2), 141-172. http://doi.org/10.1111/mila.12014

Csibra, G., \& Gergely, G. (1998). The teleological origins of mentalistic action explanations: A developmental hypothesis. Developmental Science, 1(2), 255-259. http://doi.org/10.1111/1467-7687.00039

Dörrenberg, S., Rakoczy, H., \& Liszkowski, U. (2018). How (not) to measure infant Theory of Mind: Testing the replicability and validity of four non-verbal measures. Cognitive Development. http://doi.org/10.1016/J.COGDEV.2018.01.001

Gelman, A., \& Stern, H. (2006). The Difference Between "Significant" and "Not Significant" is not Itself Statistically Significant. The American Statistician, 60(4), 328-331. http://doi.org/10.1198/000313006X152649

Havermans, R. C., Janssen, T., Giesen, J. C. A. H., Roefs, A., \& Jansen, A. (2009). Food liking, food wanting, and sensory-specific satiety. Appetite, 52(1), 222-5. http://doi.org/10.1016/j.appet.2008.09.020

Heyes, C. (2017). Apes Submentalise. Trends in Cognitive Sciences, 21(1), 1-2. http://doi.org/10.1016/j.tics.2016.11.006

Kovács, Á. M., Téglás, E., \& Endress, A. D. (2010). The social sense: susceptibility to others' beliefs in human infants and adults. Science, 330(6012), 1830-4. 
http://doi.org/10.1126/science.1190792

Krupenye, C., Kano, F., Hirata, S., Call, J., \& Tomasello, M. (2016). Great apes anticipate that other individuals will act according to false beliefs. Science, 354(6308).

Krupenye, C., Kano, F., Hirata, S., Call, J., \& Tomasello, M. (2017). A test of the submentalizing hypothesis: Apes' performance in a false belief task inanimate control. Communicative \& Integrative Biology, 10(4), e1343771. http://doi.org/10.1080/19420889.2017.1343771

Kulke, L., \& Rakoczy, H. (2018). Implicit Theory of Mind - An overview of current replications and non-replications. Data in Brief, 16, 101-104. http://doi.org/10.1016/j.dib.2017.11.016

Kulke, L., Reiß, M., Krist, H., \& Rakoczy, H. (2017). Implicit Theory of Mind across the life span Anticipatory looking data. Data in Brief, 15, 712-719. http://doi.org/10.1016/j.dib.2017.10.021

Kulke, L., von Duhn, B., Schneider, D., \& Rakoczy, H. (2018). Is Implicit Theory of Mind a Real and Robust Phenomenon? Results From a Systematic Replication Study. Psychological Science, 095679761774709. http://doi.org/10.1177/0956797617747090

Legg, E. W. (2014). A comparative approach to testing mental state attribution (Unpublished doctoral thesis). University of Cambridge, UK.

Low, J., \& Perner, J. (2012). Implicit and explicit theory of mind: State of the art. British Journal of Developmental Psychology, 30(1), 1-13. http://doi.org/10.1111/j.2044-835X.2011.02074.x

Low, \& Watts, J. (2013). Attributing False Beliefs About Object Identity Reveals a Signature Blind Spot in Humans' Efficient Mind-Reading System. Psychological Science, 24(3), 305-311. http://doi.org/10.1177/0956797612451469

Lurz, R. (2009). If chimpanzees are mindreaders, could behavioral science tell? Toward a solution of the logical problem. Philosophical Psychology, 22(3), 305-328. http://doi.org/10.1080/09515080902970673

Nichols, S., \& Stich, S. P. (2003). Mindreading: an integrated account of pretence, self-awareness, and understanding other minds. New York: Oxford University Press. http://doi.org/10.1093/0198236107.003.0003

Ostojić, L., Cheke, L. G., Shaw, R. C., Legg, E. W., \& Clayton, N. S. (2016). Desire-state attribution: Benefits of a novel paradigm using the food-sharing behavior of Eurasian jays ( Garrulus glandarius ). Communicative \& Integrative Biology, 9(2), e1134065. http://doi.org/10.1080/19420889.2015.1134065

Ostojić, L., Legg, E. W., Dits, A., Williams, N., Brecht, K. F., Mendl, M., \& Clayton, N. S. (2016). Experimenter Expectancy Bias Does Not Explain Eurasian Jays' (Garrulus glandarius) Performance in a Desire-State Attribution Task. Journal of Comparative Psychology. http://doi.org/10.1037/com0000043 
Ostojić, L., Legg, E. W., Shaw, R. C., Cheke, L. G., Mendl, M., \& Clayton, N. S. (2014). Can male Eurasian jays disengage from their own current desire to feed the female what she wants? Biology Letters, 10(3), 20140042. http://doi.org/10.1098/rsbl.2014.0042

Ostojić, L., Shaw, R. C., Cheke, L. G., \& Clayton, N. S. (2013). Evidence suggesting that desire-state attribution may govern food sharing in Eurasian jays. Proceedings of the National Academy of Sciences of the United States of America, 110(10), 4123-8. http://doi.org/10.1073/pnas.1209926110

Ruffman, T., Taumoepeau, M., \& Perkins, C. (2012). Statistical learning as a basis for social understanding in children. British Journal of Developmental Psychology, 30(1), 87-104. http://doi.org/10.1111/j.2044-835X.2011.02045.x

Schuwerk, T., Vuori, M., \& Sodian, B. (2015). Implicit and explicit Theory of Mind reasoning in autism spectrum disorders: The impact of experience. Autism, 19(4), 459-468. http://doi.org/10.1177/1362361314526004

Senju, A., Southgate, V., Snape, C., Leonard, M., \& Csibra, G. (2011). Do 18-month-olds really attribute mental states to others? A critical test. Psychological Science, 22(7), 878-80. http://doi.org/10.1177/0956797611411584

Senju, A., Southgate, V., White, S., \& Frith, U. (2009). Mindblind eyes: an absence of spontaneous theory of mind in Asperger syndrome. Science, 219(August), 883-885. http://doi.org/10.1126/science.1176170

Southgate, V., Senju, A., \& Csibra, G. (2007). Action anticipation through attribution of false belief by 2-year-olds. Psychological Science, 18(7), 587-92. http://doi.org/10.1111/j.14679280.2007.01944.x

Southgate, V., \& Vernetti, A. (2014). Belief-based action prediction in preverbal infants. Cognition, 130(1), 1-10. http://doi.org/10.1016/j.cognition.2013.08.008

Surian, L., Caldi, S., \& Sperber, D. (2007). Attribution of beliefs by 13-month-old infants. Psychological Science, 18(7), 580-6. http://doi.org/10.1111/j.1467-9280.2007.01943.x

Wellman, H. M., \& Liu, D. (2004). Scaling of theory-of-mind tasks. Child Development, 75(2), 523-41. http://doi.org/10.1111/j.1467-8624.2004.00691.x 